\title{
Cross-Cultural Adaptation, Validity and Reliability of the Arabic Version of the Falls Efficacy Scale-International (FES-I)
}

\author{
Hadeel Halaweh ${ }^{\mathrm{a}, \mathrm{b}}$ Ulla Svantesson ${ }^{\mathrm{a}}$ Susanne Rosberg ${ }^{\mathrm{a}}$ Carin Willen $^{\mathrm{a}}$ \\ aDepartment of Physiotherapy, Institute of Neuroscience and Physiology, University of Gothenburg, \\ Gothenburg, Sweden; ${ }^{b}$ Department of Physiotherapy and Rehabilitation, Health Professions College, \\ Al-Quds University, Jerusalem, Palestine
}

\section{Key Words}

Fear of falling · Falls Efficacy Scale $\cdot$ Elderly $\cdot$ Test-retest reliability

\begin{abstract}
Objective: The aim of this study was to evaluate the validity and reliability of an Arabic language version (Ar) of the Falls Efficacy Scale-International (FES-I) with respect to its use with Arabic-speaking elderly subjects. Subjects and Methods: For cross-cultural adaptation, the translation of the original English version of the scale was conducted based on the protocol of the Prevention of Falls Network Europe (ProFaNE). The FES-I (Ar) was administered via face-to-face interviews to 108 community-dwelling elderly Palestinians (61 women and 47 men, aged 60-84 years). Statistical analyses were used to determine group differences with respect to age, gender and fall history. To assess validity, Spearman's rank correlation coefficient was used to examine the correlation between the total scores of FES-I (Ar) and the Timed Up and Go (TUG) test, gait speed and balance. Testretest reliability between the two test occasions was assessed in accordance with Svensson's method. Results: The FES-I (Ar) total scores were positively correlated with TUG $\left(r_{s}=0.641, p<0.001\right)$ and negatively correlated with gait
\end{abstract}

speed $\left(r_{s}=-0.670, p<0.001\right)$ and balance $\left(r_{s}=-0.592, p<\right.$ $0.001)$. All items of the FES-I (Ar) indicated a high percentage agreement (from 88 to $93 \%$ ), and the relative position ranged from 0.01 to 0.06 . Conclusion: In this study, the FES-I (Ar) was shown to be a comprehensible, valid and reliable measure of the concern about falling among community-dwelling elderly subjects. In clinical practice and future research, the FES-I (Ar) instrument could be used to effectively assess concern about falling in Arabic-speaking elderly persons.

(c) 2015 S. Karger AG, Basel

\section{Introduction}

Worldwide, injuries in connection with falls have become a major public health problem [1]. Falls contribute to increased mortality and morbidity rates, as well as being a major cause of immobility among elderly persons $[2,3]$. The highest mortality and morbidity rates due to fall-related injuries occur in people aged 65 years and older [1]. For community-dwelling elderly people, almost half of the falls lead to an injury [2]. Fractures are the most common injuries and women have an incidence rate for fractures three times higher than men [4].

\begin{tabular}{ll}
\hline KARGER & $\begin{array}{l}\text { ( ) 2015 S. Karger AG, Basel } \\
1011-7571 / 15 / 0251-0001 \$ 39.50 / 0 \quad \text { Karger }\end{array}$ \\
$\begin{array}{l}\text { E-Mail karger@karger.com } \\
\text { www.karger.com/mpp }\end{array}$ & $\begin{array}{l}\text { This is an Open Access article licensed under the terms of the } \\
\text { Creative Commons Attribution-NonCommercial 3.0 Un- } \\
\text { ported license (CC BY-NC) (www.karger.com/OA-license), } \\
\text { applicable to the online version of the article only. Distribu- } \\
\text { tion permitted for non-commercial purposes only. }\end{array}$
\end{tabular}

Hadeel Halaweh

Institute of Neuroscience and Physiology

Department of Physiotherapy, University of Gothenburg

Box 455, SE-40530 Gothenburg (Sweden)

E-Mail hadeel.halaweh@neuro.gu.se 
The most common risk factors for falls are age and history of falls, and advanced age is an independent risk factor [5]. In the elderly, fall risk factors include impaired gait and impaired balance in combination with previous falls $[4,6]$. In addition, environmental factors seem to interact with physical abilities and are connected with an increased risk of falls in the elderly [7]. Fear of falling (FOF) and self-efficacy are psychosocial factors that impact on the incidence of falls in nonfrail adults aged 65 years and older [8].

The consequences of a fall can be more serious in elderly persons as they have greater susceptibility to injuries and the fear of another fall $[9,10]$. The reported average prevalence of FOF is $30 \%$ or more in elderly persons without a history of falling and is doubled in elderly persons who have fallen previously [9]. FOF among community-dwelling elderly persons may lead to the restriction of activity $[10,11]$. Activity avoidance or restriction due to FOF may result in social isolation and reduced quality of life, and may be a risk factor for future falls $[12,13]$. Furthermore, FOF has a long-term negative impact on physical and functional well-being, and it is a common cause of dependence among elderly people [9].

Knowledge about the variability of the level of FOF can be helpful in identifying elderly persons at risk of falling [14]. To increase this knowledge, different approaches and tools that measure FOF have been developed. In the initial research studies, single-category questions (e.g. 'In general are you afraid of falling?') were used with a 'yes/ no' or 'fear/no fear' response choice [9]. These types of questions provided limited useful information about the level of FOF [9]. In addition, these questions only addressed a generalized state of fear rather than assessing specific concerns about the possibility of falling during different activities $[9,12]$.

In this context, the Falls Efficacy Scale (FES) has been developed to assess confidence in performing 10 basic activities of daily living (e.g. cleaning the house and dressing oneself) without falling [14]. The original scale and its subsequently modified versions have been effectively used to measure FOF or fall-related efficacy among elderly persons $[12,14]$. However, the FES is most applicable with elderly individuals who are homebound and have low mobility [9]. The items of the FES refer more to basic activities and do not include more demanding or complex activities that may be relevant for higher-functioning older people [13]. In addition, the FES does not evaluate the impact of FOF on social life. For example, embarrassment, which is often a social consequence of falling, may lead to limitations of activity in an elderly person [10].
To address these issues, The FES-International (FES-I) was developed by the Prevention of Falls Network Europe (ProFaNE) [12]. The 16 items of the FES-I included the 10 original items from the FES [14] and 6 new items that assess more demanding physical activities and social activities [12, 13]. The FES-I has been used to assess FOF among elderly subjects in diverse populations and been described as a reliable and valid measure of FOF among elderly persons in different countries [12, 13, 15-18].

To develop effective and culturally sensitive health programs for Arabic-speaking elderly, researchers need a better understanding of the factors that affect survival and quality of life among the Arabic-speaking elderly. Properly addressing this issue will require reliable and valid instruments that are culturally sensitive $[19,20]$. No validated instrument exists that assesses fall-related selfefficacy among Arabic-speaking elderly persons. Therefore, the aim of this study was to evaluate the validity and reliability of an Arabic version of the FES-I with respect to its use with Arabic-speaking elderly persons.

\section{Subjects and Methods}

\section{Participants}

Community-dwelling elderly persons aged $\geq 60$ years were invited to participate in the present study. The study used the United Nations definition for age which is consistent with that used by the Palestinian Central Bureau of Statistics for the elderly and defines an old person as an individual who has reached 60 years and above [21].

The inclusion criteria were native Arabic language speakers and independence in indoor ambulation with or without walking aids (independence in indoor ambulation was determined based on the participant's walking capacity if they were able to walk independently indoors for at least $6 \mathrm{~m}$ ). Exclusion criteria were elderly participants diagnosed with severe diseases that made investigation impossible, or communication deficits (could not answer questions about their age, their children, current place, time, season and year).

In total, 108 participants were recruited from the community and from the elderly public centers in the West Bank, Palestine. All participants were informed about the aims of the study and signed to give their informed consent. Ethical approval was obtained from the research Ethics Committee of Al-Quds University, Palestine, which complies with the declaration of Helsinki.

\section{Measures}

Falls Efficacy Scale-International

The FES-I consists of 16 items that include the 10 original items from the FES and 6 more demanding items that assess walking on slippery, uneven or sloping surfaces, visiting friends or relatives, going to a social event or going to a crowded place. Falls efficacy is rated on a 4-point scale for each activity, where: $1=$ not at all concerned, $2=$ somewhat concerned, $3=$ fairly concerned and $4=$ very concerned. The total score ranged from 16 (no concern about falling) to 64 (severe concern about falling) $[12,13]$. 
Timed Up and Go

Each participant was asked to perform the Timed Up and Go (TUG) test. The test began with the participant sitting in a regular chair. When instructed, the participant stood up without using their arms, walked $3 \mathrm{~m}$, turned around, walked back to the chair and sat down again. The time it takes to complete the activity was registered in seconds. The reliability and validity of the TUG test has been established for quantifying functional mobility with older community-dwelling adults and the test is a recommended screening tool for identifying older people who are at risk for falling $[22,23]$. A cut-off value of $14 \mathrm{~s}$ was used [23].

Short Physical Performance Battery

The Short Physical Performance Battery (SPPB) includes a balance test and a 4-meter gait speed test. Each SPPB component test (balance and gait) is scored on a scale of $0-4$, with 0 indicating not attempted or could not do the test, and 4 indicating the highest category of performance. The SPPB is described as a valid and reliable measure of muscle strength and physical performance in community-dwelling older people [24].

\section{Procedure}

Cross-Cultural Adaptation of the FES-I

In this study, the cross-cultural adaptation was constructed based on the ProFaNE 10-step translation protocol [12]. The English version of the FES-I was translated from English into Arabic by two native Arabic language speakers who worked independently. These translators are health professionals, proficient English language speakers, and are familiar with the concept of FOF. A provisional local version of the FES-I Arabic (Ar) was produced via consensus with the translators. Each one of the translators selected 2 older persons to test the feasibility of the FES-I (Ar) version. Each elderly person completed the questionnaire independently and without any interruption from the translator. Next, the translator and the older person discussed the 16 items (e.g. 'Were all items clear?', 'Is it necessary to reformulate items?'). A second meeting was held to reach a consensus about a preliminary version of the FES-I (Ar). A professional translator whose native language is English performed a back translation from the Arabic language into English, and a third meeting of the initial translators was held to arrive at a consensus about the back translation. The provisional FES-I (Ar) version was reviewed by the translators considering the intentional meaning of the back translation, but not the literal meaning.

\section{Pilot Testing}

To explore whether or not all the words and phrases in the questionnaire were understandable and considered appropriate by the participants, and also to get feedback regarding any difficulty or any comment about the scale's items and response categories, the provisional version of the FES-I (Ar) was pilot tested with 10 community-dwelling elderly Palestinians (6 women and 4 men, age range 62-83 years) with different levels of education. All items and response categories in the provisional FES-I (Ar) were considered comprehensible by the participants. The piloted version was not subjected to any additional modifications and was deemed to be the final version of the FES-I (Ar).

\section{Assessment of Psychometric Properties}

The developed FES-I (Ar) was administered via face-to-face interviews. Demographic descriptive data on age, gender, living status, education level, smoking habits, medication and the presence of specific muscle diseases and/or other serious diseases were registered at the first interview. In addition, categorical questions about history of falling $[10,13]$ were asked (e.g. 'Are you afraid of falling?'). These questions included four answer alternatives: (a) no, not at all afraid; (b) yes, a little afraid; (c) yes, afraid, and (d) yes, very afraid. During the first interview, participants were also asked to perform the TUG, balance and 4-meter gait speed tests.

To evaluate the test-retest reliability, the FES-I (Ar) was readministered 7-10 days later to minimize content recall by the participants from the initial assessment or changes in events that may influence the participants concern about falling. The condition of interest was determined based on the history of falling during the test-retest interval. The same process (utilizing similar time intervals) was followed in the original work for the development and validation of the FES-I [12] and in other related studies [16, 17]. To determine whether the participants' condition of interest was stable between assessments, the participants were asked about their history of falling during the two assessments. Subjects who recorded a history of falling during the test-retest interval were excluded from the retest assessment.

\section{Statistical Analysis}

Descriptive statistics were used to characterize the sample. Between-group differences of the FES-I (Ar) total scores at the first test occasion were analyzed using the nonparametric Mann-Whitney $U$ and Kruskal-Wallis tests. Discriminate analysis of the total score of the FES-I (Ar) was conducted according to the TUG test, and a cut-off value of $14 \mathrm{~s}$ was used [23]. A p value $<0.05$ was considered significant. Data were analyzed using the Statistical Package for the Social Sciences (SPSS), version 20 (SPSS Inc., Chicago, Ill., USA).

\section{Construct Validity}

Spearman's rank correlation coefficient was used to examine the correlation between the total scores of FES-I (Ar) and TUG, gait speed, balance and FOF. Correlation coefficients $>0.70$ were considered as high, $0.50-0.69$ as moderate and $0.26-0.49$ as low [25]. In accordance with the Consensus-Based Standards for the Selection of Health Measurement Instrument (COSMIN) guidelines [26], construct validity of the FES-I (Ar) was defined to be good if $75 \%$ of our hypotheses were confirmed.

\section{Reliability}

Test-retest reliability between the two test occasions was assessed in accordance with Svensson's method [27], which is a rankbased statistical method developed to determine levels of agreement in rank invariant paired ordinal data. The percentage agreement $(\mathrm{PA})$, relative position $(\mathrm{RP})$ and relative variance $(\mathrm{RV})$ were calculated. A PA $\leq 59 \%$ was considered to be a low percentage agreement, a PA of $60-69 \%$ was viewed as moderate, and a PA $\geq 70 \%$ was interpreted as high. RP ( -1 to 1 ) was used to measure the systematic shift in categorical levels between the two assessments. Higher RP values indicate higher systematic group change, and RP values close to zero indicate negligible change over time. RV was calculated to measure the observed individual variability (the values ranged from 0 to 1 ), with higher values indicating higher individual variability [27]. For enhancing international comparisons, the internal consistency of the FES-I (Ar) was evaluated by calculating Chronbach's a coefficient for the total scores of the FES-I (Ar). 
Table 1. Demographic and clinical characteristics of the participants $(\mathrm{n}=108)$, and comparisons of the FES-I scores according to characteristic variables

\begin{tabular}{|c|c|c|c|c|}
\hline Variable & $\mathrm{n}(\%)$ & $\begin{array}{l}\text { FES-I score } \\
(\text { mean } \pm \text { SD })\end{array}$ & $\begin{array}{l}\text { Median } \\
\text { (range) }\end{array}$ & $\begin{array}{l}\mathrm{p} \\
\text { value }\end{array}$ \\
\hline \multicolumn{5}{|l|}{ Age } \\
\hline$<68$ years & $64(55.4)$ & $31.64 \pm 8.18$ & $31(16-55)$ & \multirow[t]{2}{*}{$<0.001$} \\
\hline$\geq 68$ years & $44(44.6)$ & $37.04 \pm 6.77$ & $36(23-58)$ & \\
\hline \multicolumn{5}{|l|}{ Gender } \\
\hline Female & $61(56.5)$ & $35.72 \pm 7.72$ & $34(24-58)$ & \multirow[t]{2}{*}{0.014} \\
\hline Male & $47(43.5)$ & $31.40 \pm 7.91$ & $32(16-50)$ & \\
\hline \multicolumn{5}{|l|}{ Education } \\
\hline No education & $19(17.6)$ & $38.21 \pm 8.76$ & $37(26-58)$ & \multirow[t]{4}{*}{0.014} \\
\hline Primary & $27(25.0)$ & $35.74 \pm 7.01$ & $36(24-50)$ & \\
\hline Secondary & $33(30.6)$ & $32.06 \pm 7.42$ & $32(19-48)$ & \\
\hline College & $29(26.8)$ & $31.24 \pm 7.94$ & $31(16-53)$ & \\
\hline \multicolumn{5}{|c|}{ Use of walking aids } \\
\hline No aids & $94(87.0)$ & $32.54 \pm 7.13$ & $32(16-51)$ & \multirow[t]{2}{*}{$<0.001$} \\
\hline Cane & $14(13.0)$ & $42.57 \pm 8.75$ & $42(29-58)$ & \\
\hline \multicolumn{5}{|c|}{ Afraid of falling } \\
\hline Not at all & $11(10.2)$ & $23.00 \pm 3.37$ & $23(18-29)$ & \multirow[t]{4}{*}{$<0.001$} \\
\hline A little afraid & $28(25.9)$ & $28.64 \pm 4.63$ & $29(16-37)$ & \\
\hline Afraid & $51(47.2)$ & $35.47 \pm 4.84$ & $36(24-48)$ & \\
\hline Very afraid & $18(16.7)$ & $43.94 \pm 7.90$ & $42(28-58)$ & \\
\hline \multicolumn{5}{|l|}{ Falls history } \\
\hline Yes & $33(30.6)$ & $36.93 \pm 8.50$ & $36(18-58)$ & \multirow[t]{2}{*}{0.008} \\
\hline No & $75(69.4)$ & $32.48 \pm 7.51$ & $32(16-55)$ & \\
\hline
\end{tabular}

Statistics relate to Mann-Whitney U and Kruskal-Wallis tests.

Table 2. Construct validity $(\mathrm{n}=108)$

\begin{tabular}{|c|c|c|}
\hline A priori formulated hypotheses & $\begin{array}{l}\text { Observed } \\
\text { correlation, } \\
r_{s}\end{array}$ & $\begin{array}{l}\text { Hypothesis } \\
\text { confirmed } \\
\text { (yes/no) }\end{array}$ \\
\hline $\begin{array}{l}1 \text { The FES-I (Ar) total scores and TUG } \\
\text { test are hypothesized to have a } \\
\text { positive correlation }(>0.5)\end{array}$ & 0.641 & yes \\
\hline $\begin{array}{l}2 \text { The FES-I (Ar) total scores and gait } \\
\text { speed score are hypothesized to have a } \\
\text { negative correlation }(>0.5)\end{array}$ & -0.670 & yes \\
\hline $\begin{array}{l}3 \text { The FES-I (Ar) total scores and } \\
\text { balance score are hypothesized to have } \\
\text { a negative correlation }(>0.5)\end{array}$ & -0.592 & yes \\
\hline $\begin{array}{l}4 \text { The FES-I (Ar) total scores and FOF } \\
\text { are hypothesized to have a positive } \\
\text { correlation }(>0.5)\end{array}$ & 0.759 & yes \\
\hline
\end{tabular}

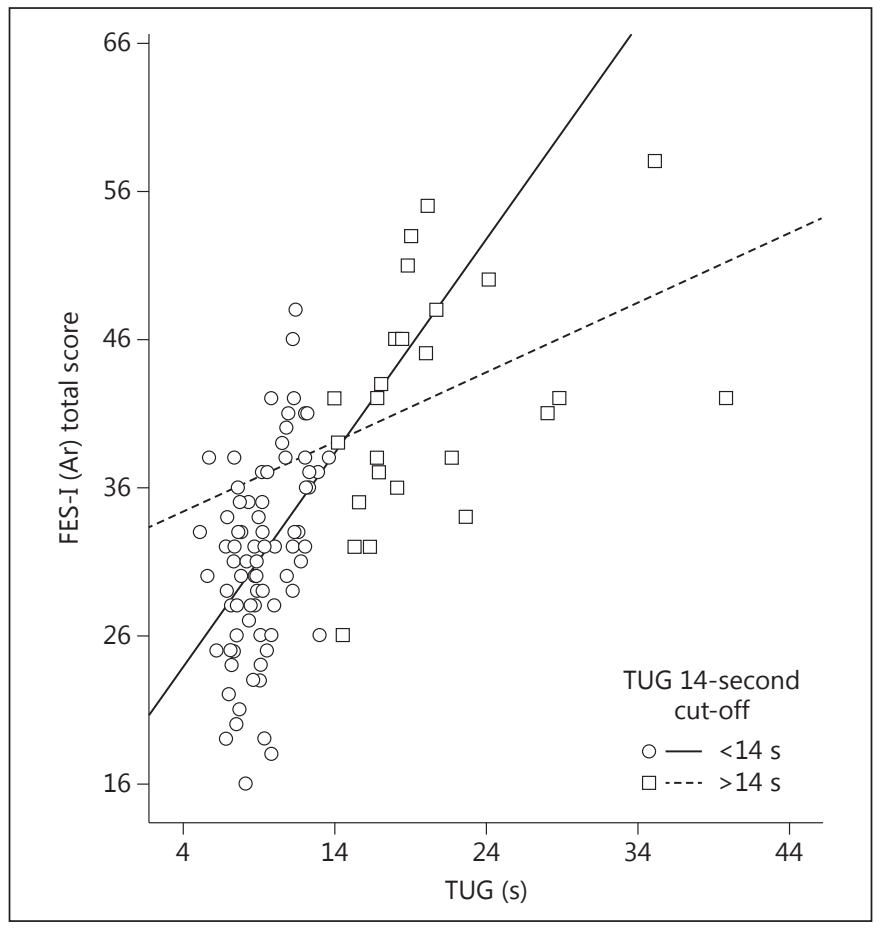

Fig. 1. Scores from the FES-I (Ar), categorized according to TUG test scores $(\mathrm{n}=108) ;<14 \mathrm{~s}: \mathrm{R}^{2}$ linear $=0.191 ;>14 \mathrm{~s}: \mathrm{R}^{2}$ linear $=$ $0.152 ; \mathrm{p}<0.001$.

\section{Results}

All the 108 (100\%) participants of this cross-cultural adaptation procedure found the FES-I (Ar) to be a comprehensible measure. The mean age of the participants was $67.3 \pm 6.10$ years. A total of $85(78.7 \%)$ participants were living with their spouse or family, 33 (30.6\%) participants had sustained one or more falls during the last 6 months, and 94 (87\%) participants were able to walk without a cane. The demographic and clinical characteristics of the participants are presented in table 1.

The total mean scores of the FES-I (Ar) were recorded as $33.84 \pm 8.06$ and $34.54 \pm 7.84$ at the first and second visits, respectively. The FES-I (Ar) scores from the first test were significantly different when divided into separate subgroups according to age, gender, education, use of walking aids, FOF and history of falling (table 1).

There was a significant difference in the FES-I (Ar) scores between the two groups according to the TUG cutoff value. Significantly higher mean scores were recorded in the FES-I ( $\mathrm{Ar}$ ) subjects who took longer than $14 \mathrm{~s}$ to complete the TUG test (42.04 \pm 7.74 compared to $31.37 \pm$ 6.37, $\mathrm{p}<0.001$; fig. 1 ). 
Table 3. Values of test-retest reliability in the FES-I (Ar) questionnaire $(n=108)$

\begin{tabular}{rlrrr}
\hline Falls & Efficacy Scale items & PA, \% & RP & RP 95\% CI \\
\hline 1 & Cleaning the house (e.g. sweeping, vacuuming or dusting) & 92 & 0.00 & -0.03 to 0.05 \\
2 & Getting dressed or undressed & 91 & 0.01 & -0.02 to 0.06 \\
3 & Preparing simple meals & 90 & 0.02 & -0.02 to 0.08 \\
4 & Taking a bath or shower & 89 & 0.02 & -0.00 to 0.06 \\
5 & Going to the shop & 93 & 0.00 & -0.03 to 0.04 \\
6 & Getting in or out of a chair & 88 & 0.02 & -0.02 to 0.07 \\
7 & Going up or down stairs & 90 & 0.06 & -0.02 to 0.10 \\
8 & Walking around in the neighborhood & 91 & 0.01 & 0.03 to 0.06 \\
9 & Reaching for something above your head or on the ground & 91 & 0.00 & -0.04 to 0.04 \\
10 & Going to answer the telephone before it stops ringing & 92 & 0.06 & 0.01 to 0.11 \\
11 & Walking on a slippery surface (e.g. wet or icy) & 90 & 0.06 & 0.02 to 0.10 \\
12 & Visiting a friend or relative & 89 & 0.04 & -0.00 to 0.09 \\
13 & Walking in a place with crowds & 91 & 0.03 & -0.01 to 0.08 \\
14 & Walking on an uneven surface (e.g. rocky ground, poorly & & & -0.01 to 0.07 \\
& maintained pavement) & 89 & 0.02 & -0.01 to 0.07 \\
15 & Walking up or down a slope & 90 & 0.02 & 0.00 to 0.11 \\
16 & Going out to a social event (e.g. religious service, family gathering & & & 0.05 \\
\cline { 2 - 4 }
\end{tabular}

Test-retest reliability is given as $\mathrm{PA}$, where $\geq 70 \%$ is considered high. Higher RP values indicate higher systematic group change.

\section{Construct Validity}

A high positive correlation $\left(\mathrm{r}_{\mathrm{s}}=0.759, \mathrm{p}<0.001\right)$ was recorded between the FES-I (Ar) total score and the responses to the categorical questionnaire about FOF. There was a moderate positive correlation between FES-I (Ar) and TUG, and moderate negative correlations were recorded between the total scores of FES-I (Ar) and the gait speed and balance scores. The results confirmed all of the a priori formulated hypotheses (table 2).

\section{Test-Retest Reliability}

All items of the FES-I (Ar) indicated a high percentage of agreement, ranging from 88 to $93 \%$. The RP ranged from 0.01 to 0.06 . Items 7,10 and 11 showed significant disagreement between test one and test two, with the recorded scores higher on the second test occasion (table 3). The values for RV were zero for all items, demonstrating no individual variability. The internal consistency (Chronbach's $\alpha$ ) of the FES-I (Ar) total score was recorded as 0.92 .

\section{Discussion}

The results of the cross-cultural adaptation of the FESI showed that the participants considered the FES-I (Ar) to be a comprehensible and appropriate instrument. The translation process was carried out taking possible cultural interpretations of different items into consideration.

The sensitivity of the FES-I in measuring both simple and more demanding physical and social activities among the elderly Palestinians of this study confirmed various results found in different cultural contexts, including in Germany, the Netherlands, the UK [13], Greece [16], Turkey [17], Sweden [18] and China [28]. In this study, the total mean score of the FES-I (Ar) was significantly higher in females than males, as well as in subjects aged above 68 years, and this finding is consistent with previous studies $[12,13,15,17]$. In addition, a significant difference in the total score of the FES-I (Ar) was seen among education levels, whereby participants with no education (illiterate) or with only a primary education had a higher mean score on the FES-I (table 1), which was comparable to a similar study [29].

Yardley et al. [12] found that the mean score of the FES-I was higher among participants who reported one fall or multiple falls in the previous year. This finding was also seen in this study, where the mean score of the FES-I was higher among participants who reported one fall or more in the previous 6 months and among participants who used walking aids (e.g. a cane). Comparable results have been reported from studies in Greece and Turkey $[16,17]$. 
In this study, the most difficult items to perform were walking on a slippery surface and walking on an uneven surface. These results are consistent with similar studies $[12,18,28]$. The recorded scores for these two new items representing more demanding activities in the FES-I indicate that there is a need for such a developed instrument compared to the original FES [14].

The mean FES-I (Ar) scores in this study were higher compared to similar studies in Greece and Turkey [16, 17] that used a self-reported mode of administration. Conversely, comparing with the results of the Chinese FES-I [28], which used the interview mode of administration, the mean scores in our study were somewhat higher. This study used interviews and recruited elderly persons with different levels of education, including subjects with no education (illiterate). Higher total mean score values have been found in studies using an interview than in studies using self-reported administration [28, 29]. A similar study [30] showed that the completion rate of questionnaires for people who were interviewed was higher than for elderly persons who completed questionnaires by themselves. The interview mode was recommended for use with frail older adults with and without cognitive impairment.

The construct validity of the FES-I (Ar) was considered to be good as all the a priori formulated hypotheses were confirmed in the present study. FOF as a single categorical question was significantly correlated with the total FES-I (Ar) score, a finding that agrees with similar studies $[13,16,17,28]$. A strong positive correlation was recorded between the FES-I total score and TUG, indicating that higher scores on the FES-I may reflect limited functional mobility among community-dwelling elderly. These findings are similar to comparable studies $[13,16$, $17,28]$. In addition, the significant difference in the scores of the FES-I (Ar) between the two groups according to the TUG analysis indicates that subjects with higher recorded FES-I scores might have a higher risk of falling. Subjects who recorded significantly higher scores on the FES-I (Ar) needed more than $14 \mathrm{~s}$ to complete the TUG test (fig. 1).

Since the FES-I is an ordinal scale, we considered using the rank-based statistical Svensson's method. The ability of Svensson's method to determine systematic group changes and individual variability merited its use for reliability testing in this study. Items 7, 10 and 11 showed significant disagreement for RP values, indicating that the recorded scores were higher on the second test occasion. This disagreement might be attributed to the fact that the items allow individual interpretations [27]. For example, some participants indicated that their concern of falling while going down stairs was more than when going up stairs (item 7). In item 10 (going to answer the telephone before it stops ringing), some responses were disputed since the availability of mobile/cell phones and handset phones no longer requires rapidity in order to answer the telephone. In addition, item 11 (walking on a slippery surface, e.g. wet or icy) may allow for individual interpretation since walking on an icy surface might be considered more difficult than walking on a wet surface. Based on these results, we recommend considering the potential individual interpretations for these items in FES-I-related studies.

To enhance the international comparison of the reliability scores, internal consistency (Chronbach's $\alpha$ ) of the FES-I (Ar) total score was calculated as 0.92. This result is comparable to the original version of the FES-I (0.96) [12] and to similar studies in Greece (0.92) [16], Turkey (0.94) [17] and China (0.94) [28]. This indicates that the results of the present study are in accordance with other related studies investigating the FES-I in different populations.

\section{Conclusion}

In this study the Arabic version of the FES-I was a comprehensible, valid and reliable instrument with which to assess concern about falling among community-dwelling elderly Palestinians. In clinical practice and future research, the FES-I (Ar) could be used to effectively assess concern about falling in Arabic-speaking elderly subjects.

\section{Acknowledgements}

We would like to thank all the participants and centers that took part in this study. We would also like to thank all the colleagues that participated in the translation process, with special thanks to Rana Shuaibi, Salam and Rabiha Abusnineh. Finally, thanks are expressed to Anna Grimby-Ekman for her contribution to the statistical analysis.

\footnotetext{
References

1 World Health Organization: Global Report on Falls Prevention in Older Age. Geneva, World Health Organization, 2008.

2 Rubenstein LZ: Falls in older people: epidemiology, risk factors and strategies for prevention. Age Ageing 2006;35:37-41.

-3 Luk J, Chan TY, Chan DK: Falls prevention in the elderly: translating evidence into practice. Hong Kong Med J 2015;21:165-171.
}

$6 \quad$ Med Princ Pract 2016;25:1-7 DOI: $10.1159 / 000441128$
Halaweh/Svantesson/Rosberg/Willen 
4 O'loughlin JL, Robitaille Y, Boivin JF, et al: Incidence of and risk factors for falls and injurious falls among the community-dwelling elderly. Am J Epidemiol 1993;137:342-354.

$\checkmark 5$ Kumar A, Carpenter H, Morris R, et al: Which factors are associated with fear of falling in community-dwelling older people? Age Ageing 2014;43:76-84.

6 Fabre JM, Ellis R, Kosma M, et al: Falls risk factors and a compendium of falls risk screening instruments. J Geriatr Phys Ther 2010;33: 184-197.

$>7$ Sattin RW, Rodriguez JG, Devito CA, et al: Home environmental hazards and the risk of fall injury events among community-dwelling older persons. J Am Geriatr Soc 1998;46: 669-676.

$>8$ Svantesson U, Babagbemi B, Foster L, et al: Influences on modern multifactorial falls prevention interventions and fear of falling in non-frail older adults: a literature review. J Clin Med Res 2014;6:314-320.

$>9$ Legters K: Fear of falling. Phys Ther 2002;82: 264-272.

10 Yardley L, Smith H: A prospective study of the relationship between feared consequences of falling and avoidance of activity in community-living older people. Gerontologist 2002;42: 17-23.

11 Stenhagen M, Ekström H, Nordell E, et al: Both deterioration and improvement in activities of daily living are related to falls: a 6-year follow-up of the general elderly population study Good Aging in Skåne. Clin Interven Aging 2014;9:1839-1846.

12 Yardley L, Beyer N, Hauer K, et al: Development and initial validation of the Falls Efficacy Scale-International (FES-I). Age Ageing 2005;34:614-619.
13 Kempen GIJM, Todd CJ, van Haastregt JCM, et al: Cross-cultural validation of the Falls Efficacy Scale International (FES-I) in older people: results from Germany, the Netherlands and the UK were satisfactory. Disabil Rehabil 2007;29:155-162.

14 Tinetti ME, Richman D, Powell L: Falls efficacy as a measure of fear of falling. J Gerontol 1990;45:P239-P243.

15 Helbostad JL, Taraldsen K, Granbo R, et al: Validation of the Falls Efficacy Scale-International in fall-prone older persons. Age Ageing 2010;39:259.

16 Billis E, Strimpakos N, Kapreli E, et al: Crosscultural validation of the Falls Efficacy Scale International (FES-I) in Greek communitydwelling older adults. Disabil Rehabil 2011; 33:1776-1784.

17 Ulus Y, Durmus D, Akyol Y, et al: Reliability and validity of the Turkish version of the Falls Efficacy Scale International (FES-I) in community-dwelling older persons. Arch Geron tol Geriatr 2012;54:429-433.

18 Nordell E, Andreasson M, Gall K, et al: Evaluating the Swedish version of the Falls Efficacy Scale-International (FES-I). Adv Physiother 2009;11:81-87.

19 Al-Jarallah K, Shehab D, Moussa MA, et al: Validation of the Arabic version of the early inflammatory arthritis detection tool. Med Princ Pract 2013;22:449-452.

20 Aburuz S, Bulatova N, Twalbeh M, et al: The validity and reliability of the Arabic version of the EQ-5D: a study from Jordan. Ann Saudi Med 2009;29:304-308.

21 Palestinian Central Bureau of Statistics (2009): Dissemination and analysis of census findings: the conditions and requirements of elderly care 1997-2007.
2 Podsiadlo D, Richardson S: The timed 'Up \& Go': a test of basic functional mobility for frail elderly persons. J Am Geriatr Soc 1991;39: 142-148.

23 Shumway-Cook A, Brauer S, Woollacott M Predicting the probability for falls in community-dwelling older adults using the Timed Up \& Go Test. Phys Ther 2000;80:896-903.

24 Mijnarends DM, Meijers JM, Halfens RJ, et al: Validity and reliability of tools to measure muscle mass, strength, and physical performance in community-dwelling older people: a systematic review. J Am Med Dir Assoc 2013;14:170-178.

25 Munro BH: Statistical Methods for Health Care Research. Philadelphia, Lippincott Williams \& Wilkins, 2005

26 Terwee CB, Bot SD, De Boer MR, et al: Quality criteria were proposed for measurement properties of health status questionnaires. J Clin Epidemiol 2007;60:34-42.

27 Svensson E: Ordinal invariant measures for individual and group changes in ordered categorical data. Stat Med 1998;17:2923-2936.

28 Kwan MMS, Tsang WWN, Close JCT, et al: Development and validation of a Chinese version of the Falls Efficacy Scale International. Arch Gerontol Geriatr 2013;56:169-174.

29 Baharlouei H, Salavati M, Akhbari B, et al: Cross-cultural validation of the Falls Efficacy Scale International (FES-I) using self-report and interview-based questionnaires among Persian-speaking elderly adults. Arch Gerontol Geriatr 2013;57:339-344.

30 Hauer K, Yardley L, Beyer N, et al: Validation of the Falls Efficacy Scale and Falls Efficacy Scale International in geriatric patients with and without cognitive impairment: results of self-report and interview-based questionnaires. Gerontology 2010;56:190-199. 